\title{
Ultrasound and microwave irradiation: contributions of alternate physicochemical activation methods to Green Chemistry
}

Received 00th January 20xx, Accepted 00th January 20xx DOI: $10.1039 / x 0 x \times 00000 x$

\author{
Gregory Chatel ${ }^{a, *}$ and Rajender S. Varma ${ }^{b, *}$ \\ This perspective article reviews the evolution of research practices and challenges reported in the literature for the use of \\ ultrasound (US) and microwave (MW) in greener chemical processes. Based on the articles published in Green Chemistry \\ journal from 1999 to now, deemed as a mirror of the evolution of these technologies in eco-compatible applications, this \\ article identifies the main thrust of research involving US and MW. Future delibrations involving these technologies by \\ themselves or in combination as well as the current and anticipated challenges are highlighted.
}

\section{Publications on ultrasound and microwave in Green Chemistry area: the evolution?}

The alternative activation methods have contributed a great deal to the evolution of methodologies that are relatively greener for organic and inorganic syntheses and transformations that have appeared in various thematic journals, especially Green Chemistry. In this perspective article, we strive to comprehend how the publications dealing with the deployment of ultrasound (US) and microwave (MW) irradiation have evolved from their first mention in Green Chemistry journal (1999) and in what way these articles have impacted the development of these technologies and consequently influenced a large population of readership as well as researchers with sustainable thinking. Indeed, the research works involving solely this kind of physicochemical activations (akin to supercritical fluids, ball-milling, nonthermal plasma, etc.) is difficult to publish in a high impact

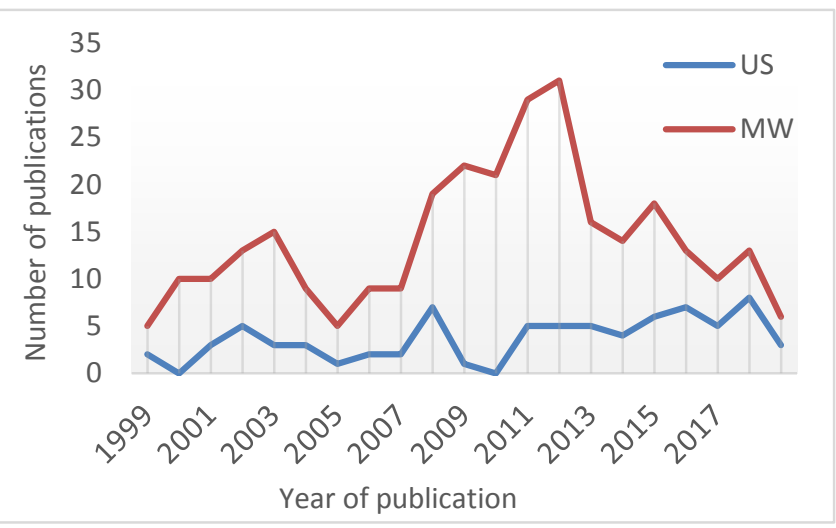

Graph 1: Evolution of the number of articles published on US and MW in Green Chemistry journal (Scopus research, September 2019).

\footnotetext{
a. Univ. Savoie Mont Blanc, LCME, 73000 Chambéry, France.

b. Regional Centre of Advanced Technologies and Materials, Department of Physical Chemistry, Faculty of Science, Palacký University in Olomouc, Šlechtitelů 27, 783
} 71 Olomouc, Czech Republic. journal such as Green Chemistry, and generally labelled as too specialized studies and often relegated to other mainstream journals with a larger audience of researchers.

We have noted that some specialized journals have been created to publish these studies in more specific communities. However, it is also prudent to widely communicate the potential of these original technologies that have transformed the classical chemistry by changing reactivities, energy usage and scientific approaches.

In the framework of the special issue on ISGC2019 conference, where a scientific session was dedicated to "Alternative Technologies", we describe herein a bibliometric analysis of the studies published specifically in Green Chemistry journal from 1999 to now, involving sonochemistry and microwave-assisted chemistry. The objectives are to illustrate the evolution of the research on US and MW themes and to identify the current scientific needs and future challenges; impediments for publication in a less specialized journal such as Green Chemistry are alluded as well.

Graph 1 depicts the evolution of the number of papers published in Green Chemistry journal from 1999 on each topical subject: sonochemistry (77 articles, average of 6 articles published each year during the last five years), microwave-assisted chemistry (297 articles, average of 14 articles published each year during the last five years) with 19 articles promoting the combination of these two technologies. In terms of the staying power in the journal, the citation rate is relatively low for a large majority of these papers (between 0 and 40 citations per articles), although some articles, highlighted in the following sections, have attained more than 1,000 citations. In addition to the targeted applications, strong demonstrations of the effects brought about by these activations can explain the interest from a larger community.

Globally, the number of publications have clearly increased, also because of the routine access to the dedicated commercial equipment in the chemical laboratories. 
However, with the routine application and adaptation of these techniques, lately, they are not often mentioned in the title or abstract which may be the reason for their apparent decline in the recent years. ${ }^{1}$

\section{Use of ultrasound for green chemistry:}

Sonochemistry is the use of ultrasound irradiation in chemical processes in a liquid medium, leading to the generation of different physicochemical effects. ${ }^{2,3}$ The intense mechanical, thermal and chemical effects of ultrasound are the consequence of the cavitation phenomenon, through the formation, the growth and the sudden collapse of gaseous microbubbles in the liquid phase. ${ }^{4,5}$ By imploding, these bubbles create locally extreme pressures (up to 1,000 bar) and high temperatures (up to 5,000 K) that can generate some interesting physical effects and initiate high-energy radical mechanisms.

One year after the publication of the twelve principles of Green Chemistry, ${ }^{6}$ Luche and Cintas described the connection between green chemistry and sonochemistry in the first issue of Green Chemistry journal (1999), a paper entitled Green chemistry: the sonochemical approach. ${ }^{7}$ This manuscript highlighted the main advantages of sonochemistry: (a) the possibility to change the course of a reaction to attain new selectivities via a "sonochemical switching"; (b) the improvement of rates, yields, and enhancement of selectivity in several examples; (c) the possibility to use non-classical reagents or reagents obtained under unusual conditions, even in aqueous media. Interestingly, other discoveries highlighted the substitution of phase transfer reagents in multiphase reactions using ultrasound. ${ }^{8}$

The interest in ionic liquids (ILs) as promising solvents, prompted Varma to emphasize that these solvents can never be green from the life cycle perspective if another solvent is used to prepare them; he pursued solvent-free preparation of ILs using ultrasound in 2002. ${ }^{9}$ During the intervening years, more than $70 \%$ of the studies published in the journal reported organic chemical reactions under US irradiation for syntheses of some ILs under ambient conditions ${ }^{10-12}$ or the preparation of organic molecules focusing on the inherent advantages to conduct synthesis in aqueous solutions, ${ }^{13,14}$ under environmentally benign conditions ${ }^{15}$ and/or with improved kinetics. ${ }^{16}$ The first US study on the preparation of nanoparticles was published in 2006 describing a simple ultrasound-assisted coating of paper with $\mathrm{ZnO}$ nanoparticles without adding any binder. ${ }^{17}$

In 2008, Varma introduced a special issue of Green Chemistry with an editorial discussing the potential of chemical activation by mechanochemical mixing, MW and US irradiation for developing cleaner processes. ${ }^{18}$

From 2012 onwards to present, although some examples from organic chemistry were still reported and not even highlighted or mentioned in the title, the keywords or in the abstract, the trend is towards the preparation of materials and (nano)catalysts involving ultrasound such as functionalized SBA-15, ${ }^{19}$ coated $\mathrm{TiO}_{2}$ or $\mathrm{ZnO}$ nanoparticles, ${ }^{20,21}$ copper oxide or graphene catalysts. ${ }^{22,23}$ The most reported effects are the depassivation of the surface, the reduction of nucleation periods and better control of crystal size without agglomeration, an improved size distribution, and the better colloidal properties. Another growth area in the recent years is the description on biomass dissolution and the extraction of organics from biomass feedstock, and pretreatment involving ultrasound. ${ }^{24-25}$ Typical examples include US- promoted depolymerization of cellulose derivatives, ${ }^{26,27}$ conversion of lignin, ${ }^{28}$ valorization of bio-waste ${ }^{29,30}$ or extractive-type processes. ${ }^{31,32}$ Highly cited article in Green Chemistry (2009) pertains to the dissolution and partial delignification of wood in ILs. ${ }^{33}$ In this study, Rogers et al. demonstrated that ultrasonic pretreatment of yellow pine sawdust for $1 \mathrm{~h}$ at $40^{\circ} \mathrm{C}$ reduced to less than half the time of complete dissolution in a chosen ionic liquid in comparison to wood without pretreatment.

Many factors can affect the cavitation phenomenon such as the frequency, the acoustic power, the gas atmosphere, the hydrostatic pressure, the nature and the temperature of the solvent, the geometry of the reactor, etc. ${ }^{34}$ The rigorous characterization of sonochemical parameters is crucial to understand the associated chemistry and facilitate the comparison between each study reported in the literature. It is important to remind here that all the sonochemical parameters and experimental conditions have to be rigorously reported in the experimental section of publications. In addition, some considerations have been made to understand and demonstrate how sonochemistry can efficiently contribute to green chemistry area in future studies. ${ }^{35}$ Importantly, the deployment of ultrasonic cleaning bath-type equipment is more an art than science as one has to have a level of water in the bath and immersion of the reacting vessel to certain depth. The inhomogeneity of their ultrasonic field could be problematic for both reproducibility and effective understanding of the phenomena occurring during US irradiation. In contrast, probe type or cup-horn equipment with good controls may be preferred for reproducibility. The design of new and specific sonochemical reactors should be one of the key parameter to help developing new processes at lab and semi-industrial scale.

\section{Use of microwaves in Green Chemistry:}

Electromagnetic radiation with frequency in the range 0.3$300 \mathrm{GHz}, \mathrm{MW}$ region, can heat matter through a dielectric mechanism that may involve dipolar polarization and ionic conduction. It is the ability of a material to absorb microwave energy and convert it into heat that causes bulk heating; the temperature of the whole sample can rise simultaneously contrary to conventional conductive heating. ${ }^{36,37}$ Even if nonthermal effects have been invoked for reactions in which the transition state is more polar than the ground state, it is generally accepted that rate enhancements largely stem from thermal effects. 38,39

Solvent-free reactions or use of benign reaction media such as polyethylene glycol and water in combination with 
the use of alternative energy-input systems, such as MW, contribute to the development of new greener reactions. ${ }^{40}$ Although MW effects have not been comprehensively explained, the MW strategy leads to time savings and achieves selectivity in organic reactions. There is continuing and contentious discussion ${ }^{41-45}$ between "specific microwave effects" that cannot be easily emulated via conventional heating means and "non-thermal microwave effects" which are proposed to explain unusual observations in $\mathrm{MW}$-assisted chemistry. ${ }^{46}$ However, a large number of examples documented in the literature, reported the advantages of MW-based systems in greener terms. ${ }^{47,48}$ In all cases, it is important to remember that $\mathrm{MW}$-assisted chemical reactions depend on the capacity of the reaction mixture to effectively absorb MW energy, which frequently depends on the polarity of reactants or ensuing intermediates, in addition to chosen solvents.

The first issue of Green Chemistry journal in 1999 had a review article from Varma entitled Solvent-free organic synthesis: using supported reagents and microwave irradiation that has been now cited more than 1250 times. ${ }^{49}$ This article summarized the activity of the solvent-free reactions under $\mathrm{MW}$ irradiation and how it can change the performance and the selectivity of the studied systems; in those days many dedicated laboratory MW systems did not exist and hence the use of open vessel chemistry on mineral supports.

From 1999 to 2003, almost exclusively, only MW-assisted organic reactions were revisited in Green Chemistry journal (an average of 10 articles/year). The proposed systems included the eco-friendly synthesis of 2-alkylated hydroquinones, ${ }^{50}$ performed in dry media for the synthesis of $\beta$-aminoalcohols, ${ }^{51}$ efficient synthesis of $\mathrm{N}$-arylamines, ${ }^{52}$ solventless for Suzuki coupling reaction on $\mathrm{Pd}$-doped alumina,53 solvent-free preparation of indazoles, pyrazolopyridines and bipyrazoles by cycloaddition reactions, ${ }^{54}$ clean oxidation of benzoins on zeolite, ${ }^{55}$ one-pot synthesis of tetrasubstituted imidazoles, ${ }^{56}$ accelerated Suzuki cross-coupling reaction in PEG, 57 among others. Remarkably, the reaction between two solids that occur below their melting points in the absence of any catalyst or a solvent, have been also demonstrated under MW. ${ }^{58,59}$ In addition, medicinal chemistry and drug synthesis are also research fields where $\mathrm{MW}$ can contribute beneficial advantages. ${ }^{60}$

Interestingly, two publications involving biomass were published in 2002 for the $\mathrm{MW}$-assisted phosphorylation of microcrystalline cellulose ${ }^{61}$ and the preparation of chelating agents from sugarcane by MW irradiation. ${ }^{62}$ In the first case, involving solvent-free system, monosubstituted phosphorous acid esters of cellulose of varying degrees of substitution of hydroxy functions (0.2-2.8) were obtained without pretreatment. ${ }^{61}$ A clear improvement in the degree of substitution of cellulose was discerned under MW (300 W, monomode system) in comparison with convention heating. The second case involved an efficient means of producing chelating agents with $14.8 \%$ of nitrogen-amide functional groups in $82 \%$ yield. ${ }^{62}$ Bagasse-derived chelating resins showed almost an identical proficiency to remove $\mathrm{Cu}$ (II) and $\mathrm{Hg}$ (II) as the commercial resin, Duolite GT-73; authors demonstrated that this method for the production of bagasse-derived chelating agents would be a viable alternative for the economic treatment of wastewater.

Fascinatingly, the next trend (from 2001) was the use of MW for the synthesis of ionic liquids (ILs) originally by Varma, initially performed in kitchen MW system while pulsing with 'on' and 'off' cycles. ${ }^{63,64}$ It was soon followed by a study under controlled conditions using a dedicated MW reactor in 2003 by Deetlefs and Seddon for the preparation of some ionic liquids. ${ }^{65}$ The MW-based methods (200 to $300 \mathrm{~W}$, multimode $\mathrm{MW}$ reactor) dramatically reduced reaction times compared to conventional methods (from several hours to 4-60 $\mathrm{min}$ ), minimized the generation of organic waste (reduction of haloalkane excesses from $10 \%$ to $1-2 \%$ ), and also afforded the ionic liquid products in excellent yields and purity. Although it was clearly pointed out in the original studies ${ }^{64}$ that being polar and ionic, ILs efficiently absorb MW energy as confirmed subsequently in studies by, Wasserscheid et al. ${ }^{66}$ and Baboulène et al. ${ }^{67}$ which promoted ILs as solvents and cosolvents in diverse applications; several investigations have been published in this area during almost one decade. 68

Rogers et al. proposed a clean, quantitative formation of methylcarbonate salts with no waste providing a platform to access large libraries of ionic liquids around common cations that are entirely halide-free, with only carbon dioxide and methanol produced as by-products through simple acid/base chemistry; $^{69}$ synthesis of 1-butyl-1-methylpyrrolidinium methylcarbonate was attained in nearly quantitative yield in only $1 \mathrm{~h}$ using microwaves.

From 2008, the use of MW heating technique shifted to the preparation of nanoparticles, including of magnetic nature. ${ }^{70-72}$ For examples, silver nanoparticles ranging from 5-10 $\mathrm{nm}$ in size were prepared in water using glutathione as benign oxidant. ${ }^{73}$ The proposed method yielded the nanoparticles within 30-60 s at a power level as low as $50 \mathrm{~W}$. Interestingly, in many cases, MW power have a beneficial effect on the morphology of synthesized nanoparticles which served as ideal nanocatalysts under quasi-homogeneous system thus bridging the good attributes of both, homogeneous and heterogeneous catalysts. ${ }^{74}$

In recent years, the dissolution of biomass and waste through the use of MW heating has been a hot topic in Green Chemistry. In this area, the fusion of ionic liquids/MW showed significant enhancement in term of dissolution of biopolymers for delignification, ${ }^{33}$ complete dissolution of lignocellulosic biomass, ${ }^{33}$ pretreatment of biomass, ${ }^{75}$ cellulose depolymerization, ${ }^{76}$ hemicellulose dissolution, ${ }^{77}$ hydrothermal treatment of biomass carbohydrates, ${ }^{78}$ to name a few. Generally, developments and applications involving biomass and $\mathrm{MW}$ are reported more frequently in the journal: extraction processes, ${ }^{79-81}$ waste valorization, ${ }^{82}$ (conversion of post-harvest tomato plant waste to levulinic acid, ${ }^{83}$ pyrolysis of waste office paper, ${ }^{84}$ hydrolysis of urban biowastes to added lignin-like products, ${ }^{85}$ valorization of fruit and vegetable by-products, ${ }^{30}$ development of citrus waste 
biorefinery, ${ }^{86}$ direct production of levoglucosenone from agricultural wastes, 87 etc.) transformation of biomass to platform molecules (conversion of carbohydrates to levulinic acid, ${ }^{88}$ conversion of polysaccharides to 5-chloromethyl furfural, ${ }^{89}$ hydrolysis of cellulose to glucose, ${ }^{90}$ production of furfural from corn stover hemicellulose, ${ }^{91}$ alcoholysis of furfural alcohol into alkyl levulinates, ${ }^{92}$ selective production of 5-hydroxymethyl furfural/levulinic acid from seaweedderived agarose, ${ }^{93}$ starch etherification and esterification, ${ }^{94}$ hydrothermal processes for the production of value-added chemicals from glycerol, ${ }^{95}$ and production of biofuels ${ }^{96}$ among others).

Finally, an innovative subject on which researchers have published article in Green Chemistry journal is the scale-up of the technology. Indeed, several examples reported the development of continuous processes under $\mathrm{MW}$ for examples for hydrodechlorination of chlorinated benzenes, 97 for the oxidation of benzyl alcohol as a model reaction, ${ }^{98}$ for the preparation of highly crystalline materials, ${ }^{99}$ or for the synthesis of self-assembled hierarchical hematite superstructures. ${ }^{100}$ Several studies involved microwaves as a heat source for carrying out various types of reactions employing circulation reaction vessels. Its use for chemical syntheses can attenuate the problem of MW heating (nonuniform heating and penetration depth) and maximize the benefits (rapid heating and first temperature adjustments). ${ }^{101}$ The process intensification involving $\mathrm{MW}$ is currently an important challenge to develop green processes on larger scales.

\section{Combination of ultrasound and microwaves:}

Table 1. Hydrazinolysis of methyl salicylate using different methods. ${ }^{103}$

\begin{tabular}{|c|c|c|c|}
\hline & $\mathrm{O}_{2} \mathrm{CH}_{3}$ & & $\mathrm{ONHNH}_{2}$ \\
\hline & $\mathrm{N}_{2} \mathrm{H}_{4} \cdot \mathrm{H}_{2} \mathrm{O}$ & & \\
\hline Entry & Method & $\begin{array}{l}\text { Reaction } \\
\text { time }\end{array}$ & $\begin{array}{l}\text { Isolated } \\
\text { yield (\%) }\end{array}$ \\
\hline 1 & Conventional reflux & $9 \mathrm{~h}$ & 73 \\
\hline 2 & $\begin{array}{l}\text { Ultrasound }(20 \mathrm{kHz}, 50 \\
\text { W) + reflux }\end{array}$ & $1.5 \mathrm{~h}$ & 79 \\
\hline 3 & $\begin{array}{l}\text { Microwave (2.45 GHz, } \\
200 \mathrm{~W})\end{array}$ & $18 \mathrm{~min}$ & 80 \\
\hline 4 & $\begin{array}{l}\text { Simultaneous MW (200 } \\
\text { W) and US (50 W) }\end{array}$ & $40 \mathrm{~s}$ & 84 \\
\hline
\end{tabular}

Interestingly, some researchers combined the effects of both, US and MW in the same system to highlight some synergetic effects. ${ }^{102}$ The first paper reported in Green Chemistry journal proposed a simultaneous $\mathrm{MW}$ and US irradiation for the synthesis of hydrazides from esters and hydrazine monohydrate. ${ }^{103}$ Table 1 reports the yield improvements between conventional reflux, US reaction, $\mathrm{MW}$-assisted reaction and combination thereof in $73,79,80$ and $84 \%$, respectively. More than an improvement in yield, this fusion of technologies allowed a drastic reduction of the reaction time from $9 \mathrm{~h}$ to $40 \mathrm{~s}$ under optimum conditions. This effect was attributed to a combination of enforced heat transfer due to $\mathrm{MW}$ irradiation and intensive mass transfer at phase interfaces due to US activation.

Cravotto and Cintas summarized the conceptual differences between MW and US (Table 2). ${ }^{104}$ They specified that the effects of these technologies often interpreted in terms of similar activation processes, but the nature of these effects are intrinsically different. The majority of the papers associated MW with a superior heating and US with an efficient agitation or mixing, but these techniques represent a great potential in term of innovation, providing additional impetus for their development in synthesis and processing. Both of them also share the inherent advantage of being green technologies, as by reducing reaction times and increasing yields they lead to an overall energy saving.

Table 2: Differences in MW and US actions as a function of application. ${ }^{104}$

\begin{tabular}{|l|l|l|}
\hline Application & US & MW \\
\hline reaction media & $\begin{array}{l}\text { aqueous and organic } \\
\text { solvents }\end{array}$ & $\begin{array}{l}\text { MW-absorbing liquids, } \\
\text { solvent-free protocols }\end{array}$ \\
\hline use of bulk metals & favorite domain & forbidden practice \\
\hline acceleration & variable (from min to h) & large (min, even s) \\
\hline activation & cavitation a still a a & thermal effects \\
\hline scaling-up & $\begin{array}{l}\text { Possible but still a } \\
\text { challenge for some } \\
\text { applications fonge for some } \\
\text { applications }\end{array}$ \\
\hline chemical effects & $\begin{array}{l}\text { selectivity changes, } \\
\text { mechanistic switching, } \\
\text { waste reductions }\end{array}$ & $\begin{array}{l}\text { selectivity changes, } \\
\text { waste reductions }\end{array}$ \\
\hline other effects & $\begin{array}{l}\text { light emission, cleaning, } \\
\text { microstreaming, } \\
\text { solubilization, etc }\end{array}$ & $\begin{array}{l}\text { heating above boiling } \\
\text { points, change in solvent } \\
\text { properties, etc. }\end{array}$ \\
\hline
\end{tabular}

However, the reproducibility still requires further attention, especially because some design parameters are sometimes overlooked. In all these cases, it is crucial to compare, separately, the silent, coupled and non-coupled conditions to highlight the synergetic effects of the combination. The synergies brought by these combinations open the door to many new applications in green chemistry where the contribution of US and MW becomes essential, although many studies on the larger scale development is imperative.

The papers combining US and MW published in Green Chemistry journal follow the trend from a majority of examples in organic chemistry (until 2006) towards progressively more applications for extraction and biomass valorization in the last ten years. In particular, the extraction of essentials oils ${ }^{105}$ or from different waste, ${ }^{106,30}$ and the valorization of glycerol ${ }^{107}$ have been investigated using the combined technology. In these explorations, low consumption of reagents, total automation, newer results and increased safety have been the main objectives. ${ }^{104}$ The reported examples demonstrate that the combination of US and $\mathrm{MW}$ can promote and improve the extraction processes for valued natural products. 


\section{Recommendations and outlooks}

We have summarized in this perspective paper that deployment of ultrasound- and microwave-irradiation, individually or in combination, have brought forth beneficial effects and innovative approaches to green chemistry. Indeed, the use of ultrasound and/or microwave is in complete agreement with the principles of green chemistry/engineering, through their numerous advantages: change of reactivity, improvement of yields and selectivity, reduction of reaction time, limitation of energy consumption and waste production, use of water/PEG as solvent instead of volatile organic solvents or solventless reactions, activation of catalysts, to name a few. The articles published in Green Chemistry journal from 1999, validate the potential of this research for developing new eco-friendly processes. Through this analysis of the literature, we also show the distinctive emerging trends in terms of applications and subject matter of research, and their evolution over the last 20 years of Green Chemistry debut. It also points out the future needs, the settlement of controversial theories, and the delineation of unknown mechanisms, thus anticipating promising years ahead for research on these two technologies.

In our opinion, when researchers want to publish on the use of US or/and MW for the benefit of a large audience and high impact journal such as Green Chemistry, it is important to propose (i) a simple, unique but not too specific system; (ii) studies and explanations on how it works (in-situ or postcharacterization of species or materials, proposition of mechanism, etc.) and can be applied to others reactions/syntheses/preparations. So, recommendation would be to focus on the following pillars: "novelty, understanding and application". In these cases, it is crucial to communicate to a large community with a didactic approach, without being too specific. It is really necessary to widely communicate the potential of these original technologies that can change the classical chemistry by altering reactivities, energy usage and scientific approaches.

Another important aspect in the area is the rigorous characterization of parameters from US and MW to understand the associated chemistry and facilitate the comparison between each study described in the literature. It is important to remind here that all the specific parameters and experimental conditions have to be rigorously reported in the experimental part of publications (frequency, powers, ultrasonic intensity, radical production, shape and geometry of the used reactors, mode of irradiation, etc.). Moreover, a systematic comparison with corresponding silent conditions (blank reaction) is required to clearly highlight the effects brought about by MW or US at specific temperatures; its optimized measurements with proper devices is very critical element for validity of the claims. Clearly, it has not been the case every time an article gets published in the literature, even in specialized journals.
Based on all this, we foresee some specific future areas of research:

(1) Finding new applications: While keeping a very rigorous approach, the newer applications will allow innovative developments (combinations of technologies, new fields of application, etc.). The analysis of articles since 1999 has shown that an innovative idea in the field often opens fresh substantial studies on these innovative appliances. Be always more innovative!

(2) Combine with other technologies: The innovation could be achieved by coupling between US/MW and other unconventional media or methods of activation. For example, use of ionic liquids in combination with ultrasound could lead to new reactivities. ${ }^{108,109}$ The combination between MW and photochemistry has shown several interesting examples where electrodeless lamps were used in $\mathrm{MW}$ reactors to speed up the chemistry. ${ }^{110}$ Sonophotocatalysis is also a field where synergetic effects could be significant. ${ }^{111,112}$ The use of the combined technology could also be interesting for a biochemical approach, for example as it was made for solid phase peptide synthesis under ultrasound irradiation. ${ }^{113}$

(3) Need for fundamental studies: The fundamental studies on sonochemistry and MW-based chemistry and their effects are essential for better understanding and recognizing the systems, delineating proposed mechanisms or demonstrate new theories. The debate, proofs of concept, and future discussions will be exciting and essential for the next generation of developed processes!

(4) Scale-up and industrial applications: The true realization of US and $\mathrm{MW}$ in green chemistry areas is dependent upon the possibility of scaling-up the excellent laboratory results for industrial uses. Some preliminary results have been encouraging with continuous or pilot scale processes, but it is essential to demonstrate the possible contribution of US and MW that open the doors to industrial applications. Scaling-up is now the challenge for these technologies!

(5) Energy issues: The overall understanding of the energy consumption for a sonochemical or MW-assisted process is essential at the laboratory scale, but even more at industrial scale. Often, the benefits brought by the technology (i.e., time savings) lead to the reduction of energy consumption. Cravotto et al. have estimated the overall energy consumption for a new pilot flow ultrasound reactor, and illustrated how to calculate the total energy consumption as exemplified in the case of biodiesel synthesis, taking into account the sum of (i) the energy to heat the oil, (ii) the energy to heat methanol and (iii) the energy to sonicate the mixture for a defined time period. ${ }^{114}$

(6) Computational calculations and modeling: We identify this track as essential, in association with fundamental studies (modeling of the phenomena under US/MW, confirmation of theories, etc.) but also the development of large-scale processes (calculations and simulations of continuous flow may help assist in the design of reactors). 
(7) Life-Cycle Assessment (LCA): To justify the greenness aspects of the developed processes, it would be imperative to determine more thoroughly the LCA of the systems to take into account more global parameters on efficiency and environment. ${ }^{115}$ The comparative LCA between classical conditions, US, MW or US/MS would be very informative as the data becomes available. ${ }^{116-119}$

\section{Acknowledgements}

GC gratefully acknowledge the Université Savoie Mont Blanc and its foundation (Chambéry, France) for their strong supports in his research activities, as well as his team, collaborators, sponsors and all contributors in this field of research. RSV thanks all collaborators for their contributions, some of whom are cited in this article.

\section{References}

1 R. B. Nasir Baig and R.S. Varma, Chem. Soc. Rev. 2012, 41, 1559-1584.

2 T. J. Mason, Sonochemistry, Oxford University Press: Oxford, 1999, 96 p.

3 G. Chatel, Sonochemistry - New opportunities for Green Chemistry, World Scientific Publishing Europe Ltd.: London, 2017, $170 \mathrm{p}$.

4 K. S. Suslick, D. A. Hammerton and D. E. Cline, J. Am. Chem. Soc. 1986, 108, 5641-5645.

5 T. J. Mason and D. Peters, Practical Sonochemistry: Power ultrasound uses and applications, Woodhead Publishing: Cambridge, 2002, $2^{\text {nd }}$ Ed., 166 p.

6 P. T. Anastas and J. C. Warner, Green Chemistry: Theory and Practice, Oxford University Press: Oxford, 1998, 1154.

7 P. Cintas and J.-L. Luche, Green Chem. 1999, 1, 115-125.

8 R. S. Varma, K.P. Naicker and D. Kumar, J. Mol. Cat. A: Chem. 1999, 149, 153-160.

9 V. V. Namboodiri and R. S. Varma, Org. Lett. 2002, 4, 3161-3163.

10 J.-M. Lévêque, J.-L Luche, C. Pétrier, R. Roux and W. Bonrath, Green Chem. 2002, 4, 357-360.

11 A. R. Gholap, K. Venkatesan, T. Daniel, R. J. Lahoti and K. V. Srinivasan, Green Chem. 2003, 5, 693-696.

12 A. R. Gholap, K. Venkatesan, T. Daniel, R. J. Lahoti and K. V. Srinivasan, Green Chem. 2004, 6, 147-150.

13 L. Yan, H. Wu and Q. Zhu, Green Chem. 2004, 6, 99-103.
14 L. Gulajski, P. Sledz, A. Lupa and K. Grela, Green Chem. 2008, 10, 279-282.

15 E. S. Sang, V. V. Yashin and A. I. Isayev, Green Chem. 2004, 6, 291-294.

16 Y. Peng, G. Song and R. Dou, Green Chem. 2005, 7, 352 356.

17 K. Ghule, A. V. Ghule, B.-J. Chen and Y.-C. Ling, Green Chem. 2006, 8, 1034-1041.

18 R. S. Varma, Green Chem. 2008, 10, 1129-1130.

19 C. Pirez, K. Wilson and A. F. Lee, Green Chem. 2014, 6, 197-202.

20 M. Jafarpour, A. Rezaeifard, M. Ghahramaninezhad and F. Feizpour, Green Chem. 2015, 17, 442-452.

21 V. Nair, J. C. Colmenares and D. Lisovytskiy, Green Chem. 2019, 21, 1241-1246.

22 P. N. Amaniampong, Q. T. Trinh, Varghese J. J., R. Behling, S. Valange, S. H. Mushrif and F. Jérôme, Green Chem. 2018, 20, 2730-2741.

23 M. Klein, A. Varvak, E. Segal, B. Markovsky, I. N. Pulidindi, N. Perkas and A. Gedanken, Green Chem. 2015, 17, 2418-2425.

24 G. Chatel, K. De Oliveira Vigier and F. Jérôme, ChemSusChem. 2014, 7, 2774-2787.

25 W. Den, V. K. Sharma, M. Lee, G. Nadadur and R. S. Varma, Frontiers Chem. 2018, 6, 141. 
26 Q. Zhang, M. Benoit, K. De Oliveira Vigier, J. Barrault, G. Jégou, M. Philippe and F. Jérôme, Green Chem. 2013, 15, 963-969.

27 F. Jérôme, G. Chatel and K. De Oliveira Vigier, Green Chem. 2016, 18, 3903-3913.

28 M. Kosa and A. J. Ragauskas, Green Chem. 2013, 15, 2070-2074.

29 S. Tabasso, D. Camaroglio, E. Calcio Gaudino and G. Cravotto, Green Chem. 2015, 17, 684-693.

30 M. Jacotet-Navarro, N. Rombaut, S. Deslis, A.-S. FabianoTexier, F.-X. Pierre, A. Billy and F. Chemat, Green Chem. 2016, 18, 3106-3115.

31 H. Passos, M. G. Freire and J. A. P. Coutinho, Green Chem. 2014, 16, 4786-4815.

32 Y. Zhang, P. Prawang, C. Li, X. Meng, Y. Zhao, H. Wang and S. Zhang, Green Chem. 2018, 20, 713-723.

33 N. Sun, M. Rahman, Y. Qin, M. L. Maxim, H. Rodriguez and R. D. Rogers, Green Chem. 2009, 11, 646-655.

34 A. Henglein and M. Gutierrez, J. Phys. Chem. 2013, 97, 158-162.

35 G. Chatel, Ultrason. Sonochem. 2018, 40, 117-122.

36 A. Loupy, Microwaves in Organic Synthesis, Wiley-VCH: $2^{\text {nd }}$ Ed., Weinheim, 2006, 990 p.

37 G. Cravotto and D. Carnaroglio, Microwave Chemistry, 2017, De Gruyter Textobook: 2017, 529 p.

38 A. de la Hoz, A. Díaz-Ortiz and A. Moreno, Chem. Soc. Rev. 2005, 34, 164-178.

39 M. B. Gawande, S. N. Shelke, R. Zboril and R. S. Varma, Acc. Chem. Res. 2014, 47, 1338-1348.

40 R. S. Varma, Green Chem. 2014, 16, 2027-2080.

41 C. O. Kappe, B. Pieber and D. Dallinger, Angew. Chem. Int. Ed. 2013, 52, 1088-1094.

42 G. B. Dudley, A. E. Stiegman and M. R. Rosana, Angew. Chem., Int. Ed. 2013, 52, 7918-7923.

43 M. R. Rosana, Y. C. Tao, A. E. Stiegman and G. B. Dudley, Chem. Sci. 2012, 3, 1240-1240.

44 C. O. Kappe, Angew. Chem., Int. Ed. 2013, 52, 7924-7928.

45 G. B. Dudley and A. E. Stiegman, Chem. Rec. 2018, 3, 381-389

46 C. O. Kappe, B. Pieber and D. Dallinger, Angew. Chem. Int. Ed., 2013, 52,1088-1094.

47 C. R. Strauss and R. S. Varma, Microwaves in Green and Sustainable Chemistry, In: Microwave Methods in Organic Chemistry, Springer: Berlin, 2006, p. 199-231.

48 R. S. Varma, Advances in Green Chemistry: chemical synthesis using microwave irradiation, Astra Zeneca Research Fondation: Bangalore, 2002
49 R. S. Varma, Green Chem. 1999, 1, 43-55.

50 H. M. Kumart Sampath, B. V. Subba Reddy, E. Jagan Reddy and J. S. Yadav, Green Chem. 1999, 1, 141-142.

51 G. Sabitha, B. V. Subba Reddy, S. Abraham and J. S. Yadav, Green Chem. 1999, 1, 251-252.

52 J. S. Yadav and B. V. Subba Reddy, Green Chem. 1999, 2, 115-116.

53 G. W. Kabalka, R. M. Pagni, L. Wang, V. Namboodiri and C. M. Hair, Green Chem. 2000, 2, 120-122.

54 J. Fraga-Dubreuil, J. R. Cherouvrier and J. P. Bazureau, Green Chem. 2000, 2, 226-229.

55 S. Balalaie, M. Golizeh and M. S. Hashtroudi, Green Chem. 2000, 2, 277-278.

56 S. Balalaie and A. Arabanian, Green Chem. 2000, 2, 274-276.

57 V. V. Namboodiri and R. S. Varma, Green Chem. 2001, 3, 146-148.

58 M. Ješelnik, R.S. Varma, S. Polanc and M. Kočevar, Green Chem. 2002, 4, 35-38.

59 M. Ješelnik, R.S. Varma, S. Polanc and M. Kočevar, Chem. Commun. 2001, 1716-1717.

60 C. O. Kappe, A. Stadler, D. Dallinger, R. Mannhold, H. Kubinyi and G. Folkers, Microwaves in Organic and Medicinal Chemistry, Wiley-VCH Verlag GmbH \& Co.: Weinheim, 2012, 683 p.

61 N. Gospodinova, A. Grelard, M. Jeannin, G. C. Chitanu, A. Carpov, V. Thiéry and T. Besson, Green Chem. 2002, 4, 220-222.

62 U. S. Orlando, A. U. Baes, W. Nishijima and M. Okada, Green Chem. 2002, 4, 555-557.

63 R. S. Varma and V. V. Namboodiri, Pure Applied Chem. 2001, 73, 1309-1313.

64 V. V. Namboodiri and R. S. Varma, Chem. Commun. 2002, 342-343.

65 M. Deetlefs and K. R. Seddon, Green Chem. 2003, 5 , 296-299.

66 J. Hoffmann, M. Nüchter, B. Ondruschka and P. Wasserscheid, Green Chem. 2003, 5, 285-290.

67 H.-P. Nguyen, H. Matondo and M. Baboulène, Green Chem. 2003, 5, 303-305.

68 G. Cravotto, L. Boffa, J.-M. Lévêque, J. Estager, M. Draye, W. Bonrath, Austr. J. Chem. 2007, 60, 946-950.

69 J. D. Hobbrey, R. D. Rogers, S. S. Shukla and C. D. Wilfred, Green Chem. 2010, 12, 407-413.

70 B. Baruwati, M. N. Nadagouda and R. S. Varma, J. Phys. Chem.C 2008, 112, 18399-18404.

71 V. Polshettiwar, B. Baruwati and R. S. Varma, ACS Nano 2009, 3, 728-736.

72 R. Luque, B. Baruwati, and R. S. Varma, Green Chem. 2010, 12, 1540-1543.

73 B. Baruwati, V. Polshettiwar and R. S. Varma, Green Chem. 2009, 11, 926-941.

74 V. Polshettiwar and R. S. Varma, Green Chem. 2010, 12, 743-754.

75 A. Zheng, Z. Zhao, K. Zhao, G. Wei, L. Jiang, X. Wang, F. He and H. Li, Green Chem. 2015, 17, 1167-1175.

76 E. G. Mission, A. T. Quitain, M. Sasaki and T. Kida, Green Chem. 2017, 19, 3831-3843. 
77 Y. Luo, J. Fan, V. L. Budarin, C. Hu and J. H. Clark, Green Chem. 2017, 19, 4889-4899.

78 J. Ramon, F. Santomauro, C. J. Chuck, A. S. Matharu and J. H. Clark, Green Chem. 2018, 20, 4507-4520.

79 H. Passos, M. G. Freire and J. A P. Coutinho, Green Chem. 2014, 16, 4786-4815.

80 A. S. Matharu, J. A. Houghton, C. Lucas-Torres and A. Moreno, Green Chem. 2016, 19, 5280-5287.

81 K. J. Viner, P. Champagne and P. G. Jessop, Green Chem. 2018, 20, 4330-4338.

82 E. Calcio Gaudino, G. Cravotto, M. Manzoli and S. Tabasso, Green Chem. 2019, 21, 1202-235.

83 S. Tabasso, E. Montoneri, D. Carnaroglio, M. Caporaso and G. Cravotto, Green Chem. 2001, 3, 302-304.

84 Z. Zhang, D. J. Macquarrie, M. De Bruyn, V. L. Budarin, A. J. Hunt, M. J. Gronnow, J. Fan, P. S. Shuttleworth and J. H. Clark, Green Chem. 2015, 17, 260-270.

85 D. Rosso, J. Fan, E. Montoneri, M. Negre, J. Clark and D. Mainero, Green Chem. 2015, 17, 3424-3435.

86 F. P. Bouxin, J. H. Clark, J. Fan and V. Budarin, Green Chem. 2019, 21, 1282-1291.

87 J. Gonzalez-Rivera, A. Spepi, C. Ferrari, C. Duce, I. Longo, D. Falconieri, A. Piras and M. R. Tiné, Green Chem. 2016, 18, 6482-6492.

88 A. Szabolcs, M. Molnar, G. Dibo and L. T. Mika, Green Chem. 2013, 15, 439-445.

89 S. W. Breeden, J. H. Clark, T. J. Farmer, D. J. MacQuarrie, J. S. Meimoun, Y. Nonne and J. E. S. J. Reid, Green Chem. 2013, 15, 72-75.

90 Y.-B. Huang and Y. Fu, Green Chem. 2013, 15, 10951111.

91 H. Comez Bernal, L. Bernazzani and A. M. Raspolli Galletti, Green Chem. 2014, 16, 3734-3740.

92 Y.-B. Huang, T. Yang, M.-C. Zhou, H. Pan and Y. Fu, Green Chem. 2016, 18, 1516-1523.

93 M. Francavilla, S. Intini, L. Luchetti and R. Luque, Green Chem. 2017, 18, 5971-5977.

94 A. Gilet, C. Quettier, V. Wiatz, H. Bricout, M. Ferreira, C. Rousseau, E. Monflier and S. Tilloy, Green Chem. 2018, 20, 2064-2074.

95 G. Zhu, J. Remon, V. L. Budarin and J. H. Clark, Green Chem. 2018, 20, 2624-2636.

96 J. Remon, J. Randall, V. L. Budarin and J. H. Clark, Green Chem. 2019, 21, 284-299.

97 U. R. Pillai, E. Sahle-Demessie and R. S. Varma, Green Chem. 2004, 6, 295-298.

98 R. J. J. Jachuck, D. K. Selvaraj and R. S. Varma, Green Chem. 2006, 8, 29-33.
99 Y.-P. Yuan, L.-S.Yin, S.-W. Cao, L.-N. Gu, G.-S. Xu, P. Du, H. Chai, Y.-S. Liao and C. Xue, Green Chem. 2014, 16, 4663-4668.

100 M. K. Bayazit, E. Cao, A. Gavriilidis and J. Tang, Green Chem. 2016, 18, 3057-3065.

101 S. Horikoshi and N. Serpone, Chem. Rec. 2019, 19, 118 139.

102 K. Martina, S. Taglieapietra, A. Barge and G. Cravotto, Top. Curr. Chem. 2016, 374, 79.

103 Y. Peng and G. Song, Green Chem. 2001, 3, 302-304.

104 G. Cravotto and P. Cintas, Chem. Eur. J. 2007, 13, 1902 1909.

105 N. Asfaw, P. Licence, A. A. Novitskii and M. Poliakoff, Green Chem. 2005, 7, 352-356.

106 J. Gonzalez-Rivera, A. Spepi, C. Ferrari, C. Duce, I. Longo, D. Falconieri, A. Piras and M. R. Tiné, Green Chem. 2016, 18, 6482-6492.

107 P. Cintas, S. Tagliapietra, E. Calcio Gaudino, E. Palmisano and G. Cravotto, Green Chem. 2014, 16, 1056-1065.

108 G. Chatel and D. MacFarlane, Chem. Soc. Rev. 2014, 43, 8132-8149.

109 G. Chatel, In: Sonochemistry: From basic principles to innovative applications, Springer: 2017, 203-223.

110 O. Pacherova, D. Chvostova, T. Kocourek, M. Jelinek, A. Dejneka, E. Eliseev, A. Morozovska and M. Tyunina, Scientific Reports, 2018, 8, 8497.

111 G. Chatel, S. Valange, R. Behling, and J.-C. Colmenares, ChemCatChem, 2017, 14, 2615-2621.

112 S. Chakma, G. Kumaravel Dinesh, S. Chakraborty and V. S. Moholkar, In: Nanophotocatalysis and Environmental Applications. Environmental Chemistry for a Sustainable World, Springer: 2020, Vol.30, p. 171194.

113 G. Wołczański, H. Płóciennik, M. Lisowski and P. Stefanowicz, Tetrahedron Lett. 2019, 60, 1814-1818.

114 P.Cintas, S. Mantegna, E. Calcio Gaudino and G. Cravotto, Ultrason. Sonochem. 2010, 17, 985-989.

115 J. H. Clark and D. J. Macquarrie, Handbook of Green Chemistry and Technology, John Wiley \& Sons: 2008, $560 \mathrm{p}$.

116 M. Cinelli, S. R. Coles, M. N. Nadagouda, J. Błaszczyński, R. Słowiński, R. S. Varma and K. Kirwan, J. Cleaner Production, 2017, 162, 938-948.

117 M. Cinelli, S. R. Coles, M. N. Nadagouda, J. Błaszczyński, R. Słowiński, R. S. Varma and K. Kirwan, Green Chem. 2015, 17, 2825-2839.

118 M. N. Nadagouda, T. Speth and R.S. Varma, Acc. Chem. Res., 2011, 44, 469-478.

119 R. Luque and R. S. Varma, Sustainable Preparation of Metal Nanoparticles-Methods and Applications, Royal Society Chemistry, RSC Green Chemistry: Cambridge, United Kingdom, 2013, 19. 